\title{
Early Experience with Serial Transverse Enteroplasty in a Korean Intestinal Rehabilitation Team
}

\author{
Jiyoon Hong, M.D. ${ }^{1}$, Sang Oh Yun, M.D. ${ }^{1}$, Sanghoon Lee, M.D. ${ }^{1,2}$, Hyun-Jung Kim, Ph.D. ${ }^{2}$, \\ Hyo Jung Park, R.Ph., M.S. ${ }^{2}$, Jeong-Meen Seo, M.D., Ph.D. ${ }^{1,2}$ \\ ${ }^{1}$ Department of Surgery and ${ }^{2}$ Intestinal Rehabilitation Team, Samsung Medical Center, Sungkyunkwan University \\ School of Medicine, Seoul, Korea
}

\begin{abstract}
Purpose: For patients with short bowel syndrome, surgery can play an important role in the management of intestinal failure (IF). Serial transverse enteroplasty (STEP) has become a popular choice of bowel lengthening among surgeons for its safety and effectiveness in improving enteral autonomy. In this study, we report 5 cases of STEP procedure on 4 patients.

Materials and Methods: In the Intestinal Rehabilitation Team at Samsung Medical Center, all patients were managed by a multidisciplinary team specialized in the care of intestinal failure patients. Retrospective review of medical records was performed.

Results: 3 patients were children and 1 patient was an adult. Improvement in enteral autonomy was achieved in all patients following each STEP procedure. Suspected suture line leakage was seen in one patient, which was successfully managed by conservative treatment There were no other serious complications related to the procedures.

Conclusion: STEP procedure continues to be an important management option in intestinal rehabilitation of patients with extreme short bowel. We report a small series of STEP performed safely and effectively in Korea. (Surg Metab Nutr 2018;9:11-15)
\end{abstract}

Key Words: Short bowel syndrome, Serial transverse enteroplasty, Intestinal rehabilitation

\section{INTRODUCTION}

Short bowel syndrome (SBS) results from extensive resection of small bowel due to various causes, including congenital anomaly in children, volvulus or thrombosis of mesenteric vessels and recurrent bowel resection conditions e.g., Crohn's disease [1] and is increasingly being recognized as a major health problem in both children and adults. Unanticipated massive bowel resection leading to SBS must be managed with long-term parenteral nutrition (PN), which is associated with low quality of life.[2]

Medical treatment of SBS includes parenteral nutrition, deliberate and patient introduction and acceleration of enteral feeding, acid blockade, elemental formulas, and potentially, the use of hormonal factors aimed at augmenting the process of intestinal adaptation.[3,4]

Surgical management of SBS includes not only the initial operative treatment of neonatal pathology, but also decisions about additional therapies such as bowel lengthening procedures or transplantation.[5-7] Factors involved in this decision include residual bowel length, degree of bowel dilation, presence of liver injury, degree of enteral tolerance, and the patient's growth curve. Serial transverse enteroplasty (STEP) was introduced in 2003 as a novel surgical technique for the treatment of patients

Received May 10, 2018. Accepted June 29, 2018.

Correspondence to: Sanghoon Lee, Department of Surgery and Intestinal Rehabilitation Team, Samsung Medical Center, Sungkyunkwan University School of Medicine, 81 Irwon-ro, Gangnam-gu, Seoul 06351, Korea

Tel: +82-2-3410-0200, Fax: +82-2-3410-0226, E-mail: sanghoone.lee@samsung.com

(c) This is an open access article distributed under the terms of the Creative Commons Attribution Non-Commercial License (http://creativecommons.org/licenses/by-nc/4.0) which permits unrestricted non-commercial use, distribution, and reproduction in any medium, provided the original work is properly cited. 
with intestinal failure secondary to SBS.[4] Many studies have correlated the ability to wean completely from parenteral nutrition with survival, highlighting the need to maximize intestinal function in these patients.[8]

In this article, we report a small series of STEP procedures performed safely and effectively in Korea.

\section{MATERIALS AND METHODS}

\section{Patients}

In the Intestinal Rehabilitation Team at Samsung Medical Center, we have performed 5 cases of STEP on 4 patients from 2012.06 to 2015.05. Retrospective review of medical records was performed and last outside clinic follow up was 2018.05.

In order of our experience, we reviewed demographic data such as gender, age, primary disease, operation time and day as well as other patient characteristics related to the cause and severity of the SBS. Treatment and outcome of the patients' intestinal rehabilitation were also outlined.

STEP procedure is indicated when intestinal failure is caused by SBS and bowel caliber is greater than $4 \mathrm{~cm}$ on imaging study using contrast. We used Endo-GIA (Medtronics, Minnesota, USA) $30 \mathrm{~mm}$ or $45 \mathrm{~mm}$ (tan, purple) with $2 \mathrm{~cm}$ intervals on either sides of the bowel and did not perform reinforcement sutures (Fig. 1).
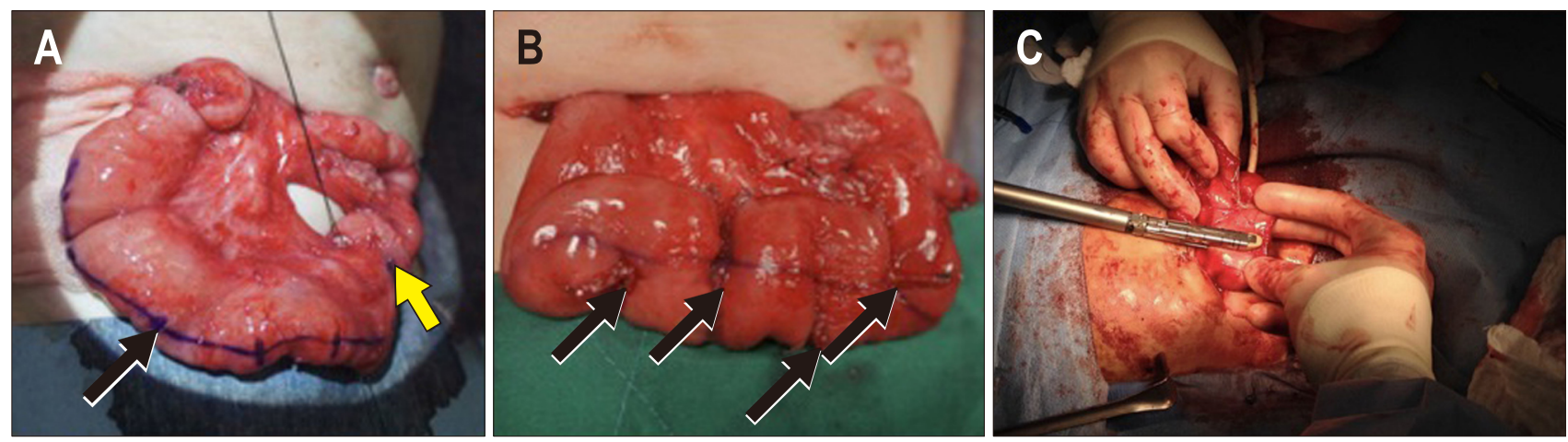

Fig. 1. Patient 1 operation field. (A) Previous STEP procedure bowel state. Black arrow: dilated small bowel which is much reduced state compared with previous operation. Yellow arrow head: small bowel - colon anastomosis site on previous operation. (B) Stapler site with Endo GIA. (C) Operator is doing stapling on STEP procedure.

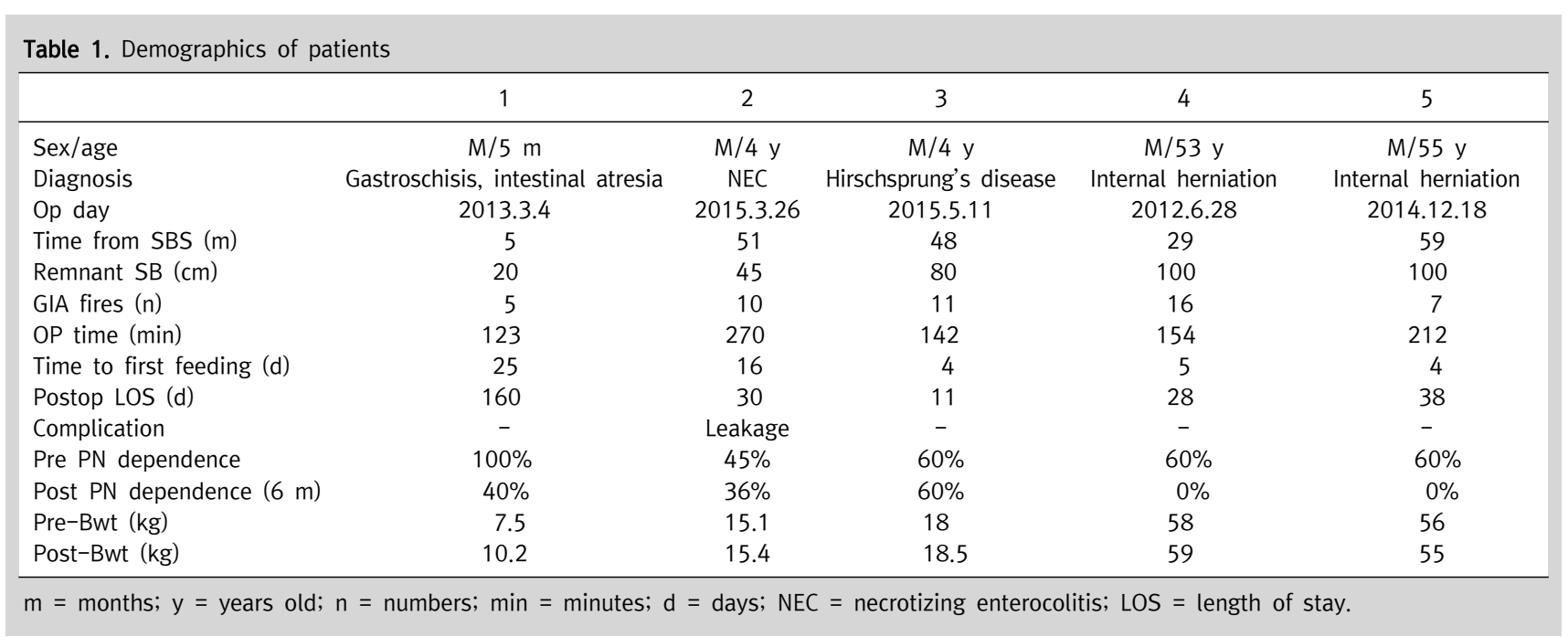




\section{RESULTS}

Three patients were children and one patient was an adult. Patient 1 was a 26 month-old boy with short bowel syndrome due to gastroschisis and jejunal atresia. STEP procedure was performed at 6 months after birth. Patient 2 was an 8-year-old boy with short bowel syndrome due to total colonic aganglionosis. STEP procedure was performed at 4 years old. Patient 3 was a 6-year-old boy with short bowel syndrome due to necrotizing enterocolitis. STEP procedure was performed at 4 years old age. Patient 4 was a 58-year-old male with short bowel syndrome due to internal herniation after gastrectomy. STEP procedure was performed 3 years after the occurrence of short bowel and then a second STEP procedure was performed after 4 years from first STEP. Demographic data of the patients undergone STEP are outlined in Table 1.

Four out of the five cases showed decreased PN dependence (\%) before and 6 months after STEP procedure. Adult patient cases were weaned off $\mathrm{PN}$ at postoperative 6 months following STEP (Fig. 2A). In the adult cases, so many recurrent central line-associated bloodstream infection (CLABSI) was occurred that central line could not be kept any longer. So we believed that STEP could improve intestinal rehabilitation and that could wean off $\mathrm{PN}$ which means no more need central line.

Three pediatric cases showed weight gain during the 6-month postoperative period following STEP (Fig. 2B).
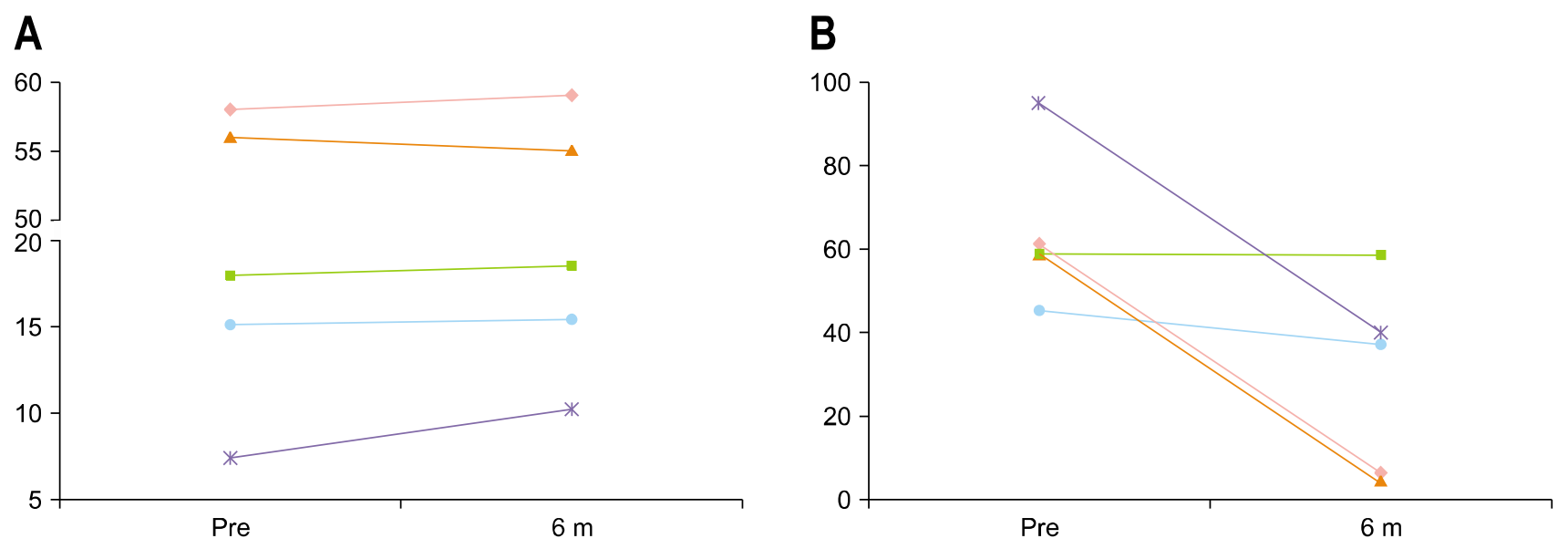

Fig. 2. (A) PN dependence change before and after STEP procedure. (B) Weight change before and after STEP procedure.
Assessment of patients' nutritional status was done by serial analyses of hemoglobin, albumin, prealbumin and total lymphocyte counts. Hemoglobin levels increased 6 months after STEP compared to preoperative levels (Fig. 3A). Albumin and prealbumin levels also increased steadily after STEP (Fig. 3B and 3C). Total lymphocyte counts peaked at postoperative 3 months and remained stable during the following 12 months (Fig. 3D).

Minor leakage was suspected in one patent who had taken multiple bowel surgeries because the patient showed unusual abdomen pain and tenderness was seen. Lab resulted mild leukocytosis so we did conservative treatment that of 7 days of antibiotics treatment which was successful. There were no other serious complications related to the procedures.

\section{DISCUSSION}

Short bowel syndrome is a devastating condition that is characterized clinically by an inability to absorb adequate enteral nutrition to sustain normal growth and development in children or sustain body weight in adults. A variety of conditions can cause short bowel syndrome leading to loss of normal bowel mucosal surface area [8] but recently the management of SBS was improved with the effective application of PN.[9,10] The use of PN permits the survival of patients who would have otherwise died of the complications of malnutrition and it gives

\footnotetext{
Fig. 2. (A) PN dependence change before and after STEP procedure. (B) Weight change before and after STEP procedure.
} 

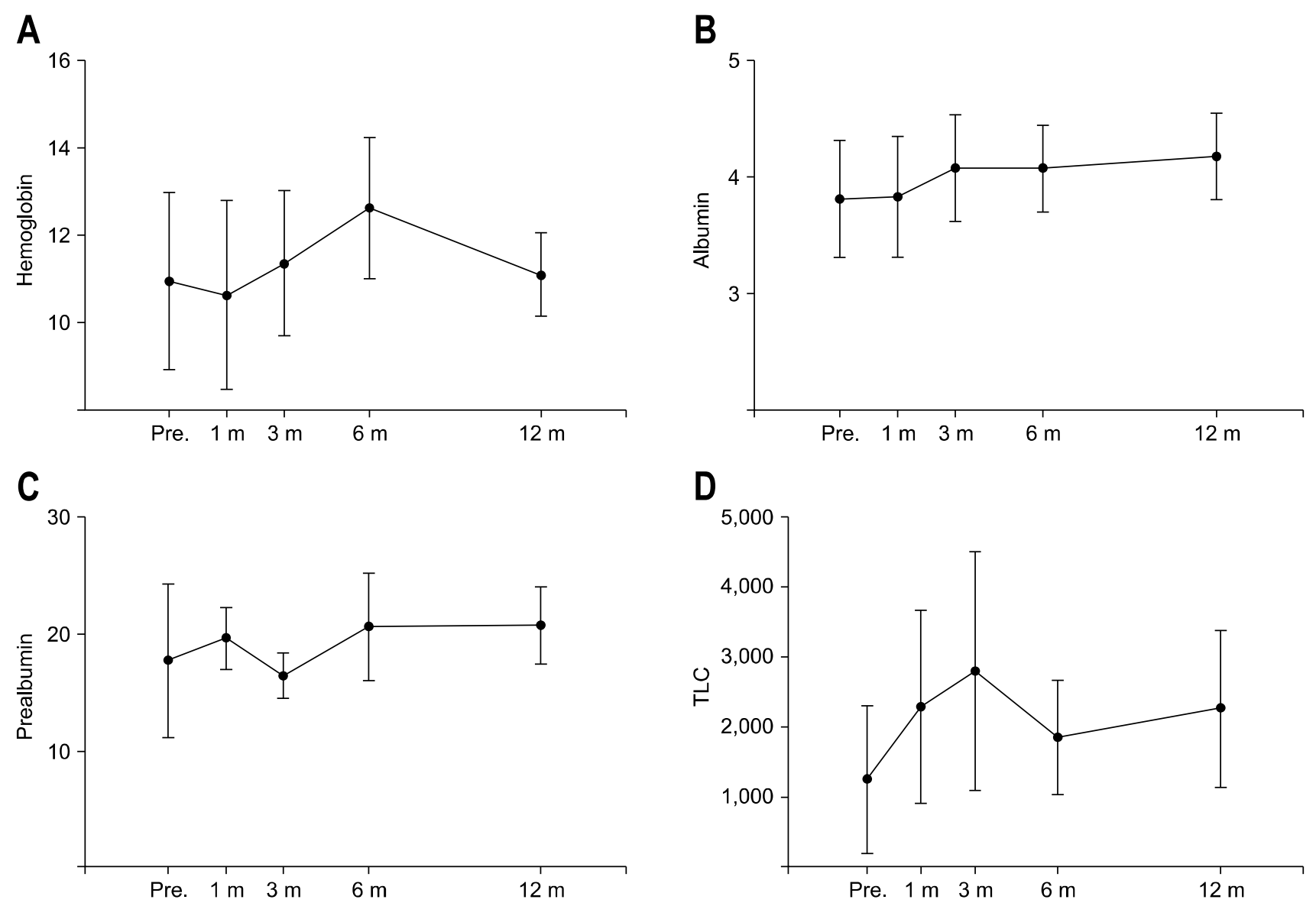

Fig. 3. (A) Hemoglobin change before and after STEP procedure. (B) Albumin level change before and after STEP procedure. (C) Prealbumin level change before and after STEP procedure. (D) Total lymphocyte level change before and after STEP procedure.

times to patients to adapt until full enteral feeding is possible. The process of bowel adaptation includes mucosal hyperplasia, which results in increased mucosal surface area.

However, not all patients survive through PN management and we must reduce the dependence of PN for the patients' improved quality of life. For that reason, we performed STEP procedure in refractory cases despite maximal medical management including PN formula personalization, cyclic feeding and bacterial overgrowth treatment.[11]

STEP procedure for SBS is aimed at correcting two anatomic problems, which are length and diameter. Bianchi [12], Kimura and Soper [13] have described in their studies on the concept of bowel lengthening in SBS. The concept of "splits" is described by Bianchi which is based on the principle that each section of bowel is supplied by individual blood supply by the mesentery to the bowel split when it enters into the bowel wall. So mesenteric blood supply can be maintained to each half of the bowel after being divided longitudinally. The procedure described by Kimura and Soper is based on allowing time for the antimesenteric border of the bowel to "parasitize" a new blood supply from the liver and abdominal wall musculature before dividing it from the mesenteric half and creating a new tube. Both concepts aim to double the bowel length and reduce its circumference. By reducing the bowel diameter, we can improve the stasis of bowel, bacterial overgrowth and mucosal inflammation caused by dilatation. $[9,11]$

Short term outcomes of the STEP procedure have been shown to be safe and effective in many reports.[1,2] However, long term outcomes of the STEP procedure have been difficult to evaluate because STEP is just one 
aspect of the multidisciplinary management of SBS known as intestinal rehabilitation. Moreover, intestinal rehabilitation is both rapidly changing and different from center to center. Many studies have shown good long-term outcomes after STEP procedure in SBS patients. Modi et al. [4] also showed mean increase in length of dilated bowel segment of $89 \% \pm 26 \%$ and increase in total small bowel length of $46 \% \pm 40 \%$. Seven patients in their study were weaned off PN at 4 years. Carlsson et al. [2] reported large quantity of data on STEP procedure from International Serial Transverse Enteroplasty (STEP) Data Registry that showed $47 \%$ of patients attaining full enteral tolerance following STEP. In our center, we aimed to for patients to returned to normal life most of all, weight gain and nutritional balance are also considered for patients follow up evaluation.

In our report, there are several limitations. Data were collected from only one center and the number of case is small which means that we could not generalize the outcome of STEP procedure and its effectiveness in the treatment of SBS. The causes of SBS of each patient were different which means general condition of each patient is different and it could affect the outcome of variables such as PN dependence, body weight change and the other nutritional status. We compared PN dependence for pre and post operation after 6 months, and this cannot show whole evaluation. For example, patient 3 showed no change in PN dependence in 6 months however, 3 years later, the patient gained weight and have tried to decreased dependence at outpatient clinic.

In conclusion, STEP is a novel procedure for SBS patients' refractory to medical treatment or for reducing PN dependency to maintain good quality of life. We per- formed 5 STEP procedures in 4 children and adults with SBS as a part of their intestinal rehabilitation and all of the patients showed stable nutritional parameters during 12 months' follow-up with considerable decrease in PN dependence.

\section{REFERENCES}

1. Thompson JS. Comparison of massive vs. repeated resection leading to short bowel syndrome. J Gastrointest Surg 2000;4: 101-4.

2. Carlsson E, Bosaeus I, Nordgren S. What concerns subjects with inflammatory bowel disease and an ileostomy? Scand J Gastroenterol 2003;38:978-84.

3. Goulet O, Ruemmele F. Causes and management of intestinal failure in children. Gastroenterology 2006;130(2 Suppl 1):S16-28.

4. Modi BP, Javid PJ, Jaksic T, Piper H, Langer M, Duggan C, et al. First report of the international serial transverse enteroplasty data registry: indications, efficacy, and complications. J Am Coll Surg 2007;204:365-71.

5. Georgeson K, Halpin D, Figueroa R, Vincente Y, Hardin W Jr. Sequential intestinal lengthening procedures for refractory short bowel syndrome. J Pediatr Surg 1994;29:316-20; discussion 320-1.

6. Thompson JS. Edgar J. Poth Memorial Lecture. Surgical aspects of the short-bowel syndrome. Am J Surg 1995;170: 532-6.

7. Thompson JS, Langnas AN, Pinch LW, Kaufman S, Quigley EM, Vanderhoof JA. Surgical approach to short-bowel syndrome. Experience in a population of 160 patients. Ann Surg 1995; 222:600-5; discussion 605-7.

8. Andorsky DJ, Lund DP, Lillehei CW, Jaksic T, Dicanzio J, Richardson DS, et al. Nutritional and other postoperative management of neonates with short bowel syndrome correlates with clinical outcomes. J Pediatr 2001;139:27-33.

9. Kim HB, Fauza D, Garza J, Oh JT, Nurko S, Jaksic T. Serial transverse enteroplasty (STEP): a novel bowel lengthening procedure. J Pediatr Surg 2003;38:425-9.

10. Dudrick SJ, Wilmore DW, Vars HM, Rhoads JE. Long-term total parenteral nutrition with growth, development, and positive nitrogen balance. Surgery 1968;64:134-42.

11. Mercer DF, Hobson BD, Gerhardt BK, Grant WJ, Vargas LM, Langnas AN, et al. Serial transverse enteroplasty allows children with short bowel to wean from parenteral nutrition. J Pediatr 2014;164:93-8.

12. Bianchi A. Intestinal loop lengthening--a technique for increasing small intestinal length. J Pediatr Surg 1980;15:145-51.

13. Kimura K, Soper RT. A new bowel elongation technique for the short-bowel syndrome using the isolated bowel segment lowa models. J Pediatr Surg 1993;28:792-4. 